\title{
Routine Preoperative Hematocrit Testing in Adults Presenting for Day Case Surgery: is It Necessary in A Developing Environment
}

\author{
*Ilori, Iniabasi $\mathrm{U}^{1}$, Eyo, Catherine $\mathrm{S}^{2}$ \\ ${ }^{I}$ Department Of Anaesthesiology, University Of Calabar Teaching Hospital, Calabar, Nigeria \\ ${ }^{2}$ Department Of Anaesthesia, University Of Uyo Teaching Hospital, Uyo, Nigeria
}

\begin{abstract}
Background: Preoperative hematocrit estimation has been one of the basic routine requirements before an anaesthetic is administered. Non availability of hematocrit result or a value less than $30 \%$ often led to cancellation of an elective surgery by the anaesthetist.

Objective: The aim of the study was to determine the prevalence of anemia among adult patients presenting for day case surgery in two Nigerian Tertiary Hospitals.

Patients and method: The hematocrit levels of patients, 16 years or above, who presented for ambulatory surgery at two tertiary healthcare facilities in Southern Nigeria over a three year period were retrospectively examined for prevalence of anemia. The data was extracted from anesthetic records. Anemia was taken as hematocrit level of less than $30 \%$.

Results: A total of 423 patients had their surgeries done on day case basis during the period. There were 245 females and 178 males. There was no significant difference between the mean hematocrit of the males (40.99\%) and that of the females $(36.56 \%)(P>0.9)$. Age showed significant correlation with the hematocrit decreasing with age in males $(P=0.002)$ and increasing with age in the females $(P=0.048)$. The overall prevalence of anemia was $2.6 \%$.

Conclusion: Preoperative anemia in Nigerian adult patients presenting for day case surgery showed a low prevalence. Preoperative hematocrit evaluation in healthy adult patient presenting for day case surgery should be based on clinical findings, the magnitude of contemplated surgery and anticipated blood loss rather than as a routine.
\end{abstract}

Keywords: Adult patients, ambulatory surgery, anemia, developing countries, low prevalence

\section{Introduction}

Preanesthetic testing of any organ function is aimed at detecting or confirming an abnormality in order to guide perioperative interventions by providing the baseline value. [1] Preoperative hematocrit or hemoglobin estimation has been one of the routine requirements in all patients before an anaesthetic. Routine hematocrit or hemoglobin estimation prior to anesthesia and surgery was necessary because of tissue oxygen supply in the surgical patient. The prevalence of anemia taken as a hematocrit level of $30 \%$ or hemoglobin of $10 \mathrm{~g} / \mathrm{dl}$, has been noted to be rare in healthy adults. [2] In the West African sub-region, patients are prone to malaria, malnutrition and other infestation that tend to cause anemia. This study was aimed at establishing the prevalence of anemia (hematocrit level of $<30 \%$ ) in adult patients presenting for day case surgeries.

\section{Patients And Method}

The hematocrit level of adult patients who presented for ambulatory surgeries at two tertiary hospitals in South-South Region of Nigeria over a three year period was retrospectively examined for prevalence of anemia. Exclusion criteria included gynecological surgeries and patients with any hematological disorder. The data that was extracted from anesthetic records included patient's age, sex, hematocrit level and surgery done. Analysis of the data was done using simple descriptive statistics with Microsoft 2010 Excel worksheet. A Student $t$ - test was used to compare variables between male and females. A T-test value of 0.05 was taken as significant. Anemia was taken as hematocrit level of less than $30 \%$.

\section{Results}

A total of 423 patients were operated on day case basis during the period. These were 274(64.78\%) excision biopsies of superficial lumps, 59(13.95\%) herniorraphies, 44(10.4\%) varicocoelectomies and $46(10.87 \%)$ other minor surgeries. There were 178 males and 245 females giving a male to female ratio of 1 : 1.38. There was a significant difference in the mean ages of the males, $36.39 \pm 14.33$ years, (range $16-82$ years) and that of the female patients $31.43 \pm 12.88$ years, (range $16-76$ years) with a $P$ value of 0.0003 . Seventy-five percent $(75.1 \%)$ of the females (184) and $68 \%$ of the males (117) were below 40 years of age. There was no significant difference between the mean hematocrit of all the males $40.99 \pm 4.71 \%($ range $=27-54)$ and that of 
all the females $36.56 \pm 3.94 \%$ (range $26-49)(\mathrm{P}>0.9)$. Table 1 is a comparison of the mean hematocrit in different age groups in decade, between the males and females. There was significant difference in the hematocrit level between the males and the females in age group 31 to 60years. Age showed significant correlation with the hematocrit. For males it was a negative correlation with a $\mathrm{P}$ value of 0.002 indicating that the hematocrit decreases as the age increases. The converse was for the females where the hematocrit increases with age, $\mathrm{P}=0.048$.

Table 1: Age Comparison of hematocrit between males and Females

\begin{tabular}{|c|c|c|c|}
\hline $\begin{array}{l}\text { Age Group } \\
\text { (years) }\end{array}$ & $\begin{array}{l}\text { Mean Hematocrit Females } \\
\text { (Frequency) }\end{array}$ & $\begin{array}{l}\text { Mean Hematocrit } \\
\text { males (Frequency) }\end{array}$ & P Value \\
\hline$\leq 20$ & $35.96(50)$ & $40.33(21)$ & 0.0004 \\
\hline $21-30$ & $36.40(101)$ & $42.21(52)$ & 5.15 \\
\hline $31-40$ & $37.93(40)$ & $41.26(54)$ & 0.0005 \\
\hline $41-50$ & $36.88(32)$ & $40.94(17)$ & 0.01 \\
\hline $51-60$ & $36.67(15)$ & $41.58(19)$ & 0.002 \\
\hline $61-70$ & $37.25(4)$ & $36.46(13)$ & 0.7 \\
\hline$>70$ & $30.00(3)$ & $33.00(2)$ & 0.27 \\
\hline
\end{tabular}

Nine $(3.7 \%)$ females and two $(1.1 \%)$ males had their hematocrit less than $30 \%$ giving an overall prevalence of anemia as $2.6 \%$.

\section{Discussions}

Determination of hematocrit (HCT) prior to any surgery has become an integral part of pre-anesthetic evaluation. The sole objective is to ensure adequate supply of oxygen to all the vital organs. [1] Anemia is defined by the World Health Organization as hemoglobin concentration of less than $(<) 13 \mathrm{~g} / \mathrm{dl}(\mathrm{HCT}=40 \%)$ in males and $<12 \mathrm{~g} / \mathrm{dl}(\mathrm{HCT}=37 \%)$ in females. [3] There is association between the severity of anemia as an independent factor and perioperative adverse outcome. [4-6] The concept of transfusion trigger resulted in the recommendation of minimum acceptable hemoglobin level of $10 \mathrm{~g} / \mathrm{dl}$ corresponding to a hematocrit level of $30 \%$ before surgery. This has been widely accepted as the 10/30 rule. [7] The reported perioperative prevalence of anemia in surgical patients varies widely from $5 \%$ to $76 \%$ and depends on the patient's disease and comorbidities, the surgical procedure and associated blood loss, and the definition of anemia used. [8] Preoperative anemia taken as hemoglobin level of $<10 \mathrm{~g} / \mathrm{dl}$ or hematocrit level of less than $30 \%$ in healthy adults has been reported to be rare .[2,7] In our study of healthy adults presenting for day case surgery, the prevalence of anemia was $2.6 \%$. Olson et al in a study of patients presenting for outpatient surgery, reported a prevalence of $0.8 \%$. The lower prevalence could be that they used a hemoglobin level of less than $9 \mathrm{~g} / \mathrm{dl}$ to indicate anemia.[2] Nze PU[9] in a review of 500 outpatient surgeries used a hemoglobin level of less than $10 \mathrm{~g} / \mathrm{dl}$ and reported a prevalence of $6 \%$. Pal et al [10] in Pakistan retrospectively studied 320 patients that had general surgical procedures including emergencies reported eight females and one male in otherwise healthy patients that had their hemoglobin below $10 \mathrm{~g} / \mathrm{dl}$ giving a prevalence of $2.8 \%$. This is similar to the finding in this study where nine females and two males had their hematocrit less than $30 \%$ with a prevalence of $2.6 \%$. Other researchers used the WHO definition of anemia to determine their hemoglobin cut- off level with varying prevalence of anemia ranging from $12 \%$ in general surgical patients to $35 \%$ in patients undergoing orthopedic surgery [11-13]. These authors reported that more females where anemic than the males. This was a similar finding in this study were the prevalence anemia was higher in females, 3.7\%, though the average hematocrit level was above $30 \%(36.56 \%)$ than the $1.1 \%$ in males. About $81.82 \%$ (9 out of 11$)$ of the patients that had hematocrit level less than $30 \%$ were females. Nze PU similarly reported that $80 \%$ of the patient that were anemic were females. [9] This might be attributed to the fact that $75 \%$ of the females were below $40 y e a r s$ which is within the reproductive range.

It was on this premise that gynecological cases were excluded as bleeding is often the commonest presentation. However, age showed a significant correlation in both males and females. In the males the hematocrit decreases with increasing age while in the females it was an increase with increasing age. A similar findings was reported among American adults from a study of mean blood hematocrit. The study also reported that all age groups studied except 18 -24years, the mean hematocrit was higher in the males than that of the females. [14] This is also similar to this study and is seen in Table 1 where the hematocrit in males was higher than that of the females in all the age groups. Between the ages of 30 and 60 years and below 20 years, the hematocrit of the males were significantly higher than the females.

The used of hemoglobin level of $10 \mathrm{~g} / \mathrm{dl}$ or hematocrit of $30 \%$ was to indicate when to transfuse. This level, though taken as moderate anemia by WHO standard, however, arbitrarily using a hemoglobin or hematocrit level to indicate anemia has been challenged. Wedgwood et al [7] in their review documented that in 
clinical practice, functional disturbance and morbidity associated with metabolic demand with a given level of hemoglobin is more important than an arbitrary definition of normality. Carson et al in their study of patients that declined blood transfusion reported that though mortality was inversely related to the preoperative hemoglobin, however no patient with a hemoglobin level above $8 \mathrm{~g} / \mathrm{dl}$ and operative blood loss below $500 \mathrm{ml}$ was reported to have died.[5]

The value of preoperative hematocrit estimation in healthy adults undergoing surgery with minimum blood loss has been questioned. This is because minor abnormalities detected often in apparently healthy adults do not influence perioperative management or outcome in patients who are undergoing surgery with minimal blood loss. [1,11 15 -17] Johnson et al in their study of 212 adult patients undergoing a variety of ambulatory surgical procedures reported that abnormality in laboratory tests did not influence perioperative cancellations, intraoperative or postoperative complications, or admissions to the hospital from the day case unit after the surgical procedures [15]. Chung et al[16] in their study, randomized day case surgical patients to either indicate testing or no testing. They showed that there were no significant differences in the rates of perioperative adverse events and 30-day hospital re-visits between patients who underwent the indicated testing versus those with no preoperative testing before day case surgery. There was also no perioperative mortality. They all observed that the decision regarding a patient's fitness for surgery may be accurately predicted on the basis of history and clinical examination. In this study all the patients that had their hematocrit below $30 \%$ all had their surgery without any adverse effect or blood transfusion in that the blood loss was minimal. Moreover none of the patient studied had their hematocrit below 26\%. This is in agreement with Carson et al findings that no patient with hemoglobin above $8 \mathrm{~g} / \mathrm{dl}$ or hematocrit of $24 \%$ died. [5] Preoperative testing should therefore generally be directed by history and physical examination. The relevance of any tests should be considered in light of the type of procedure that is planned, particularly the hemodynamic changes and blood loss involved. [18,19]. In our malaria, sickle cell disease and various bloodletting parasitic infestations that may be prevalent, the prevalence of anemia is low. Nevertheless, screening for anemia and determination of hemoglobin or hematocrit level prior to any surgery has been an integral part of preanesthetic evaluation in many healthcare institutions especially in third world countries including ours. The rationale is more of a custom than valid scientific evidence. [19] Many professional Associations have given guidelines on preoperative testing in order to reduce unnecessary tests and the cost of healthcare. There are evidences that perioperative risk does not seem to rise until the hemoglobin level falls below $8 \mathrm{~g} / \mathrm{dl}$ or hematocrit of 24\%). [5,7] Preoperative hematocrit estimation should therefore be based on clinical suspicion, co- existing morbidities and anticipated perioperative blood loss rather than as a routine pre-anesthetic requirement.

\section{Conclusion}

The prevalence of anemia in Nigerian adults presenting for day case anesthesia is low even in females. Laboratory testing should not be substituted for clinical history and physical examination. Preoperative hematocrit estimation should therefore be based on clinical suspicion, co- existing morbidities and anticipated perioperative blood loss rather than as a routine pre-anesthetic requirement even in underserve environment.

\section{References}

[1]. Kotur, PF. Is it necessary to estimate haemoglobin level routinely prior to surgery? Indian Journal of Anaesthesia, 50(2), 2006, 148 $-149$

[2]. Olson RP, Stone A, Lubarsky D. The Prevalence and Significance of Low Preoperative Hemoglobin in ASA 1 or 2 Outpatient Surgery Candidates. Anesth Analg 101, 2005,1337-1340

[3]. Haemoglobin concentrations for the diagnosis of anaemia and assessment of severity. World Health organisation 2011 WHO/NMH/NHD/MNM/11.1

[4]. Goodnough LT, Dubois, RW, Nissenson AR, Anemia: Not Just an Innocent Bystander? Arch Intern Med,163(23), 2003, 1400 1404

[5]. Carson JL, Spence RK, Poses RM, Bonavita G. Severity of Anaemia and Operative Mortality and Morbidity. Lancet 2, 1988 , 727 729

[6]. Baron DM, Hochrieser H, Posch M, Metnitz B, Rhodes A, Moreno RP et al. Preoperative anaemia is associated with poor clinical outcome in non-cardiac surgery patients. British Journal of Anaesthesia 2014, 1 - 8 doi:10.1093/bja/aeu098

[7]. Wedgwood JJ, Thomas JG. Peri-operative haemoglobin: an overview of current opinion regarding the acceptable level of haemoglobin in the peri-operative period. European Journal of Anesthesiology,13, 1996, 316-324.

[8]. Shander A, Knight K, Thurer R, Adamson J, Spence R. Prevalence and outcomes of anemia in surgery: a systematic review of the literature. Am J Med, 116(suppl 7A), 2004,58S-69S.

[9]. Nze PU. The utility of pre-operative haemoglobin testing in adult day-case surgical patients. The Tropical Journal of Health Sciences, 13 (2), 2006, 39-41.

[10]. Pal KMI, Khan IAR, Safdar B. Preoperative Work Up: Are the Requirements Different in A Developing Country? JPMA, 48, $1998,339-341$,

[11]. Johnson RK, Mortimer AJ. Routine pre-operative blood testing: is it necessary? Anaesthesia 57, 2002 914-917

[12]. Khan S, Khan MU, Samad S. Can Simple Preoperative Hemoglobin Testing Screen Symptomatic Anemia in Patients Undergoing Ambulatory Surgeries in Third World Countries? Open Journal of Anesthesiology, 2, 2012,150-153 
[13]. Goodnough LT, Maniatis A, Earnshaw P, Benoni G, Beris P, Bisbe E. Detection, evaluation, and management of preoperative anaemia in the elective orthopaedic surgical patient: NATA guidelines. British Journal of Anaesthesia, 106 (1), 2011, 13-22

[14]. Stewart WH, Gardner JW. Mean blood haematocrit of adults; United States 19620 - 1962. Public Health publications, no 1000, 1967, series 11-No. 24 downloaded from www.cdc.gov/nchs/data/series/sr_11/sr11_024acc.

[15]. Johnson H Jr, Knee-Ioli S, Butler TA, Munoz E, Wise L. Are routine preoperative laboratory screening tests necessary to evaluate ambulatory surgical patients? Surgery,104 (4), 1988, 639-645.

[16]. Chung F, Yuan H, Yin L, Vairavanathan S, Wong DT. Elimination of Preoperative Testing in Ambulatory Surgery. Anesth Analg $108,2009,467-475$

[17]. Turnbull JM, Buck C. The value of preoperative screening investigations in otherwise healthy individuals. Arch Intern Med, 147(6), 1987, 1101-1105.

[18]. Hesse S, Seebauer A, Schwender D. Ambulatory anesthesia: which preoperative screening tests are required? Anaesthesist, 48(2), 1999, 108-115.

[19]. Hepner DL The role of testing in the preoperative evaluation. Cleveland Clinic Journal of Medicine 76(Supp 4), 2006, S 22 - S27

[20]. Munro J, Booth A, Nicholl J Routine preoperative testing: a systematic review of the evidence. Health Technology Assessment, 1: No. 12,1997 